\title{
Prospects for detecting dark matter halo substructure with pulsar timing
}

\author{
Shant Baghram, ${ }^{1, *}$ Niayesh Afshordi, ${ }^{2,3}$ and Kathryn M. Zurek ${ }^{4}$ \\ ${ }^{1}$ Department of Physics, Sharif University of Technology, P.O. Box 11365-9161, Tehran, Iran \\ ${ }^{2}$ Perimeter Institute for Theoretical Physics, 31 Caroline St. N., Waterloo, ON, N2L 2Y5, Canada \\ ${ }^{3}$ Department of Physics and Astronomy, University of Waterloo, 200 University Avenue West, Waterloo, ON, N2L 3G1, Canada \\ ${ }^{4}$ Michigan Center for Theoretical Physics, Department of Physics, University of Michigan, Ann Arbor, Michigan 48109, USA
}

(Received 27 January 2011; published 10 August 2011)

\begin{abstract}
One of the open questions of modern cosmology is the nature and properties of the dark matter halo and its substructures. In this work we study the gravitational effect of dark matter substructures on pulsar timing observations. Since millisecond pulsars are stable and accurate emitters, they have been proposed as plausible astrophysical tools to probe the gravitational effects of dark matter structures. We study this effect on pulsar timing through Shapiro time delay (or integrated Sachs-Wolfe (ISW) effect) and Doppler effects statistically, showing that the latter dominates the signal. For this task, we relate the power spectrum of pulsar frequency change to the matter power spectrum on small scales, which we compute using the stable clustering hypothesis, as well as other models of nonlinear structure formation. We compare this power spectrum with the reach of current and future observations of pulsar timing designed for gravitational wave detection. Our results show that while current observations are unable to detect these signals, the sensitivity of the upcoming square kilometer array is only a factor of few weaker than our optimistic predictions.
\end{abstract}

DOI: 10.1103/PhysRevD.84.043511

PACS numbers: 95.35.+d, 98.80.-k

\section{INTRODUCTION}

One of the greatest puzzles of modern cosmology is the nature of the dark matter (DM). The latest cosmological observations indicate that DM has a mean cosmic mass density $\sim 5$ times larger than the density of the baryonic matter (e.g. see WMAP-7 yr results [1]), and its presence is confirmed by a large amount of astrophysical evidence, such as rotation curves of galaxies, gravitational lensing effects, growth of large scale structure of the Universe, big bang nucleosynthesis and the dynamics of the Universe as a whole [2]. The cosmological and astrophysical observations that may lead us to better understand this unknown component of the Universe are areas of intense study, focusing on DM's nature as particles, its structure, distribution and effect on the other components of the Universe. Studying the small scale structure of DM, for example, will tell us something about the DM particle's fundamental properties. Consequently, finding new footprints of DM in astrophysical observations is important for opening new horizons in DM studies.

The theory of structure formation, which is based on gravitational instability of primordial matter density fluctuations and the hierarchal scheme of structure formation, assumes that collisionless DM is the main ingredient in today's cosmological structures. One of the features of this theory is that DM collapses into bound states, known as DM halos. A cold dark matter primordial power spectrum predicts a large range of mass scales for these DM halos, from $10^{12}-10^{14} M_{\odot}$ down to $10^{-12}-10^{-4} M_{\odot}$ [3]. Larger

\footnotetext{
*baghram@physics.sharif.edu
}

halos form from merger of smaller halos which may partly survive as substructure of bigger halos.

The statistics of DM distribution and the dynamics of this substructure may have an effect on astrophysical observations. One of the promising astrophysical probes for studying the distribution of interstellar medium (ISM) which is considered to be mostly baryonic matter are pulsars [4]. ISM causes a dispersion on pulsars' light which in turn has an effect on pulsar timing residuals.

In the present work, we push this one step further and study the gravitational effects of dark matter halo substructure on pulsar timing. This effect manifests itself through the (1) Shapiro time-delay effect [5], and (2) Doppler effect. The Shapiro time-delay is caused by the presence of dark matter's dynamical potential along the line of sight. On the other hand, the Doppler effect is caused by the acceleration of the observer/pulsar because of the pull of DM subhalos. Probing dark matter substructure by Shapiro time delay in pulsar timing was first proposed by Siegel et al. by considering the effect of one DM subhalo crossing the line of sight [6]. Dark matter studies with pulsar timing continued by Seto and Cooray [7], Pshirkov et al. [8] and recently by Ishiyama et al. [9]. On the other hand the Shapiro delay effect was studied for relativistic neutrino and photons of SN1987A [10] and also for low frequency pulsars in globular cluster [11]. It is worth mentioning that pulsar timing was also used to study other astronomical effects [12], as a recent example the effect of DM subhalos crossing the line of sight was studied in astrometric microlensing [13].

In this work, as a complementary and more realistic view, we consider the statistical distribution of DM 
substructure and its effect on pulsars' timing residual. In order to study the effect of the DM substructure distribution on pulsar timing, we need a structure formation model. On very small scales deep into the nonlinear regime of structure formation, which is unaffected by halo merging or tidal disruption, we can use the stable clustering hypothesis. The stable clustering hypothesis was first introduced by Davis and Peebles [14] as an analytic technique to study the galaxy correlation function in the deeply nonlinear regime, and was subsequently applied to fitting formulas for nonlinear correlation functions/power spectra $[15,16]$. In the current work, we use the phase-space stable clustering model which was recently developed by Afshordi et al. [17].

The article is structured as follows. In Sec. II, we first introduce millisecond pulsars. Then in the following subsections we derive the power spectrum of frequency change of pulsars for Shapiro time delay and Doppler effects. In Sec. III, we review the stable clustering hypothesis in phase space. In Sec. IV, we find the frequency change power spectrum and show its dependence on free parameters of the model, both for Shapiro and Doppler effects. In Sec. V, we discuss the observational prospects of detecting these effects with current and future pulsar timing arrays. Finally, Sec. VI concludes the paper.

For reference, we set cosmological parameters to be $\Omega_{m}^{0}=0.27, \sigma_{8}=0.8$ and $H_{0}=100 h \mathrm{~km} / \mathrm{s} / \mathrm{Mpc}$ where $h=0.7$.

\section{GRAVITATIONAL EFFECT ON POWER SPECTRUM OF PULSAR TIMING}

In this section, we first introduce millisecond pulsars as promising astrophysical observational probes to detect the gravitational effects of DM substructures. Then we derive the statistics of frequency change due to Shapiro and Doppler effects.

\section{A. Millisecond pulsars}

The most stable, consistent astrophysical emitters in the known universe are millisecond pulsars, many of them remaining stable without flux change over time scales exceeding 30 years [18]. On account of this they have been used as precise tools to probe changes in the matter distribution between the pulsar and earth [19]. The pulsars with the highest rotational frequencies, and hence the shortest pulse to pulse periods, are the most stable with a time period of $\mathcal{O}(1 \mathrm{~ms})$. The typical residual of these pulsars is of order of $\mathcal{O}(1 \mu \mathrm{s})$. This means that fluctuations in pulsar period within a short time scale (e.g. $\sim 1 \mathrm{hr}$ ) are less than $\sim \mu \mathrm{s}$. These residuals do not accumulate, which means that the period remains constant during the time that a pulsar is stable. This is used to measure the pulsar's timing residuals with high accuracy during a long period $(\sim 10$ years $)$, and to search for nonintrinsic changes in pulsar timing. Consequently, to detect any physics besides the pulsars' intrinsic changes, we should search for a time delay larger than the intrinsic uncertainties. An important point is that many interesting nonintrinsic effects on pulsar timing will be correlated. An example is the attempt to detect gravitational waves through cross-correlation of pulsar timing arrays [20]. Another possible nonintrinsic effect which we consider in this work is the change of the gravitational potential. The transit of DM halo substructure across the line of sight, which causes the Shapiro delay, is studied in the following subsection. This discussion is followed by a consideration of the Doppler effect, caused by the acceleration of pulsar/observer due to presence of DM substructure.

\section{B. Shapiro time delay}

The Shapiro time delay is caused by the presence of a time dependent gravitational potential along the line of sight. To quantify this effect, we can write the metric of perturbed space time as

$$
d s^{2}=-(1+2 \Phi) d t^{2}+(1-2 \Phi) d \vec{x}^{2},
$$

where $\Phi$ represents the Newtonian potential in the weak field limit and we set the speed of light $c=1$. In the case of pulsars, we can write the null geodesics for a pulse received at time $t$ using the above metric as

$$
t=t_{0}+\delta t=\int_{x_{\mathrm{em}}}^{x_{\mathrm{obs}}}(1-2 \Phi) d \vec{x},
$$

where $\delta t$ is obtained from integration over the perturbed potential along the line of sight. As it is not possible to measure the absolute light travel time of any astrophysical object, we need to observe the time arrival changes over a detection period. The time derivative of the pulsar time residual is defined as

$$
\dot{\delta} t=-\frac{\delta \nu}{\nu}=-2 \int \dot{\Phi} d \vec{x},
$$

where $\nu$ is the frequency of the pulsar, and $\delta \nu$ is the change in frequency (we note that this is identical to the cosmological integrated Sachs-Wolfe (ISW) effect [21]). In order to find a statistical description of the induced time delays, we assume that DM substructures move with a constant velocity $v$ across the line of sight in the $x$-direction. We refer to this as the moving screen approximation, which enables us to relate the time derivatives to the spatial gradients of metric, i.e. $\dot{\Phi}=v \frac{\partial}{\partial x} \Phi$. The temporal correlation of the frequency changes is then given by

$$
\left\langle\left(\frac{\delta \nu}{\nu}\right)_{l}\left(\frac{\delta \nu}{\nu}\right)_{l l}\right\rangle=4 \int_{0}^{z_{0}} \int_{0}^{z_{0}} d z_{l} d z_{l l} v^{2}\left\langle\frac{\partial}{\partial x} \Phi_{l} \frac{\partial}{\partial_{x}} \Phi_{l l}\right\rangle,
$$

where $z_{0}$ is the position of the pulsar in the $z$-direction (line of sight), which we take to be $\sim 1 \mathrm{kpc}$, and $\Phi_{l}$ and $\Phi_{l}$ correspond to potentials at two different times. Now we 
can express the right-hand side of Eq. (4) in Fourier space as:

$$
\begin{aligned}
\left\langle\left(\frac{\delta \nu}{\nu}\right)_{l}\left(\frac{\delta \nu}{\nu}\right)_{l l}\right\rangle= & 4 v^{2} \int_{0}^{z_{0}} \int_{0}^{z_{0}} d z_{l} d z_{l l} \int \frac{d^{3} \vec{k}_{l}}{(2 \pi)^{3}} \\
& \times \int \frac{d^{3} \vec{k}_{l l}}{(2 \pi)^{3}}\left(i k_{l}^{x}\right)\left(i k_{l l}^{x}\right)\left\langle\Phi\left(\vec{k}_{l}\right) \Phi\left(\vec{k}_{l l}\right)\right\rangle \\
& \times e^{-i \vec{k}_{l} \cdot \vec{r}_{l}} e^{-i \vec{k}_{l l} \cdot \vec{r}_{l l}} .
\end{aligned}
$$

By integrating over $z_{l}$ and $z_{l l}$, and using the definition of the potential power spectrum,

$$
\left\langle\Phi\left(\vec{k}_{l}\right) \Phi\left(\vec{k}_{l}\right)\right\rangle=(2 \pi)^{3} \delta^{3}\left(\vec{k}_{l}+\vec{k}_{l}\right) P_{\Phi}(\vec{k}) .
$$

Equation (5) becomes

$$
\begin{aligned}
\left\langle\left(\frac{\delta \nu}{\nu}\right)_{l}\left(\frac{\delta \nu}{\nu}\right)_{l l}\right\rangle= & 4 v^{2} \int \frac{d^{3} k_{l}}{(2 \pi)^{3}}\left[k_{l}^{x} z_{0} \operatorname{sinc}\left(\frac{k_{l}^{z} z_{0}}{2}\right)\right]^{2} \\
& \times P_{\Phi}\left(\vec{k}_{l}\right) e^{-i k_{l}^{x}\left(x_{l}-x_{u}\right)}
\end{aligned}
$$

where $\operatorname{sinc}(x) \equiv \frac{\sin (x)}{x}$. We can take the integral over $k^{z}$, which results in

$$
\begin{aligned}
\left\langle\left(\frac{\delta \nu}{\nu}\right)_{l}\left(\frac{\delta \nu}{\nu}\right)_{l l}\right\rangle= & 4 z_{0} v^{2} \iint \frac{d k_{y}}{2 \pi} \frac{d k_{x}}{2 \pi} \\
& \times P_{\phi}(\vec{k}) \times\left(k_{x}\right)^{2} e^{-i k^{x} v\left(t_{1}-t_{2}\right)},
\end{aligned}
$$

where we omit the subscript $l$ and replace $x_{l}=v t_{l}$ in the moving screen approximation (we also assume $k_{z} \sim$ $\left.z_{0}^{-1} \ll k_{x}, k_{y}\right)$. Now by considering the definition of the time-delay power spectrum,

$$
\left\langle\left(\frac{\delta \nu}{\nu}\right)_{l}\left(\frac{\delta \nu}{\nu}\right)_{l l}\right\rangle=\frac{1}{2 \pi} \int P_{((\delta \nu) /(\nu))}(\omega) e^{-i \omega \Delta t} d \omega,
$$

we can integrate Eq. (8) over the time difference of two observations to obtain the power-spectrum,

$$
\begin{aligned}
P_{((\delta \nu) /(\nu))}(\omega)= & \int\left\langle\left(\frac{\delta \nu}{\nu}\right)_{l}\left(\frac{\delta \nu}{\nu}\right)_{l l}\right\rangle^{i \omega \Delta t} d(\Delta t) \\
= & \int d(\Delta t) 4 z_{0} v^{2} \iint \frac{d k_{y}}{2 \pi} \frac{d k_{x}}{2 \pi} \\
& \times P_{\phi}(\vec{k})\left(k_{x}\right)^{2} e^{-i k^{x} v(\Delta t)} e^{i \omega \Delta t},
\end{aligned}
$$

where $\Delta t=t_{1}-t_{2}$. Now the integration over $d k_{x}$ and $d(\Delta t)$ gives us the relation between $k_{x}$ and the frequency as $k_{x} v=\omega$. Consequently Eq. (10) simplifies to

$$
\omega P_{((\delta \nu) /(\nu))}(\omega)=\frac{4 z_{0}}{v} \int \frac{d k^{y}}{2 \pi} \omega^{3} P_{\phi}\left(\sqrt{\frac{\omega^{2}}{v^{2}}+k_{y}^{2}}\right) .
$$

Using the Poisson equation, we can relate the potential power spectrum to the matter power spectrum $P_{\rho}(\vec{k})$ :

$$
P_{\Phi}(\vec{k})=\left(\frac{4 \pi G}{k^{2}}\right)^{2} P_{\rho}(\vec{k}) .
$$

Finally by inserting Eq. (12) in Eq. (11), we find the dimensionless $\omega P(\omega)$ in terms of the matter power spectrum:

$$
\begin{aligned}
& \left.\omega P_{((\delta \nu) /(\nu))}(\omega)\right|_{\text {Shapiro }} \\
& \quad=\frac{4 z_{0}}{v} \int \frac{d k_{y}}{2 \pi} \omega^{3}\left(\frac{4 \pi G}{k^{2}}\right)^{2} \bar{\rho}^{2} P_{\mathrm{NL}}\left(\sqrt{\frac{\omega^{2}}{v^{2}}+k_{y}^{2}}\right),
\end{aligned}
$$

where we replace $P_{\rho}(\vec{k})=\bar{\rho}^{2} P_{\mathrm{NL}}(\vec{k})$, in which $\bar{\rho}$ is the mean cosmic DM density. In Sec. IV, we will derive this function by using the stable clustering hypothesis.

\section{Doppler effect}

Changing the potential of DM substructure near pulsars or the Earth will introduce a velocity shift, which affects pulsar frequencies via the Doppler effect. In the Doppler effect, the frequency change of a pulsar is related to the line of sight velocity as $\frac{\delta \nu}{\nu}=v_{l . s .}$. So the correlation of the frequency changes observed at two separate times $t_{1}$ and $t_{2}$ caused by the Doppler effect can be written as

$$
\begin{aligned}
\left\langle\left(\frac{\delta \nu}{\nu}\right)_{l}\left(\frac{\delta \nu}{\nu}\right)_{l l}\right\rangle= & \int_{-\infty}^{t_{1}} d t \int_{-\infty}^{t_{2}} d t^{\prime}\left\langle\nabla_{z} \Phi_{l} \nabla_{z} \Phi_{l l}\right\rangle \\
& \times e^{\varepsilon\left(t-t_{1}\right)} e^{\varepsilon\left(t^{\prime}-t_{2}\right)},
\end{aligned}
$$

where $\varepsilon$ is a small parameter to regulate the infrared divergence of the integral. Once more, we can write the right-hand side of Eq. (14) in Fourier space. Integration over time variables with the limit of $\varepsilon \rightarrow 0$ results in

$$
\left\langle\left(\frac{\delta \nu}{\nu}\right)_{l}\left(\frac{\delta \nu}{\nu}\right)_{l l}\right\rangle=\int \frac{d^{3} \vec{k}}{(2 \pi)^{3}} P_{\phi}(\vec{k}) \frac{\left(k_{z}\right)^{2}}{\left(k_{x} v\right)^{2}} e^{-i k^{x} v(\Delta t)},
$$

where we used the moving screen approximation to replace time integrals by integrals over $x$. Again, using Eq. (9), we can write the power spectrum of frequency changes as

$$
\begin{aligned}
P_{((\delta \nu) /(\nu))}(\omega) & =\int\left\langle\left(\frac{\delta \nu}{\nu}\right)_{l}\left(\frac{\delta \nu}{\nu}\right)_{l l}\right\rangle^{i \omega \Delta t} d(\Delta t) \\
& =\int d(\Delta t) \int \frac{d^{3} \vec{k}}{(2 \pi)^{3}} P_{\phi}(\vec{k}) \frac{\left(k_{z}\right)^{2}}{\left(k_{x} v\right)^{2}} e^{-i k^{x} v(\Delta t)} e^{i \omega \Delta t} .
\end{aligned}
$$

Integration over $d k_{x}$ and $d \Delta t$ gives us the relation between $k_{x}$ and the frequency as $\omega=k_{x} v$. Consequently Eq. (16) results in

$$
P_{((\delta \nu) /(\nu))}(\omega)=\frac{1}{v} \int \frac{d k_{y}}{2 \pi} \int \frac{d k_{z}}{2 \pi} \frac{k_{z}^{2}}{\omega^{2}} P_{\Phi}(\vec{k}) .
$$

Because of the symmetry between the integration over $k_{y}$ and $k_{z}$, we can replace $k_{y}^{2}$ by $\left(k_{y}^{2}+k_{z}^{2}\right) / 2$ in Eq. (17), which leads to 


$$
P_{((\delta \nu) /(\nu))}(\omega)=\frac{1}{4 \pi} \int d k_{*} \frac{k_{*}^{3}}{v \omega^{2}} P_{\phi}\left(\sqrt{\frac{\omega^{2}}{v^{2}}+k_{*}^{2}}\right)
$$

where $k_{*}=\sqrt{k_{y}^{2}+k_{z}^{2}}$. By using the Poisson equation, we can relate the potential power spectrum to matter power spectrum, and finally write the dimensionless power spectrum as

$$
\begin{aligned}
& \left.\omega P_{((\delta \nu) /(\nu))}(\omega)\right|_{\text {Doppler }} \\
& \quad=\frac{1}{4 \pi} \int d k_{*} \frac{k_{*}^{3}}{v \omega}\left(\frac{4 \pi G}{k^{2}}\right)^{2} \bar{\rho}^{2} P_{\mathrm{NL}}\left(\sqrt{\frac{\omega^{2}}{v^{2}}+k_{*}^{2}}\right) .
\end{aligned}
$$

As in the case of Shapiro delay, by knowing the matter power spectrum we can determine the pulsar timing power spectrum.

\section{STABLE CLUSTERING HYPOTHESIS}

In this section, we discuss the stable clustering hypothesis as a model to describe the nonlinear structure formation that will give us the required matter power spectrum. We make use of the phase-space stable clustering model recently developed by Afshordi et al. [17]. The collisionless Boltzmann equation at the phase-space coordinates, $\vec{r}+\Delta \vec{r}, \vec{v}+\Delta \vec{v}$ is approximately given by

$$
\begin{aligned}
& \frac{d f}{d t}(r+\Delta r, v+\Delta v, t) \\
& \quad \simeq \frac{\partial f}{\partial t}+\frac{\partial f}{\partial r} \cdot(v+\Delta v)-\frac{\partial f}{\partial v} \cdot[\nabla \Phi+(\Delta r \cdot \nabla) \nabla \Phi] \\
& \quad=0
\end{aligned}
$$

where $\Phi$ is the gravitational potential, and for simplicity we omit the vector signs of distances and velocities. We can reexpress the above equation in terms of the phasespace density in the comoving coordinates with particle $i$ :

$$
\tilde{f}_{i}(\Delta r, \Delta v) \equiv f\left(r_{i}+\Delta r, v_{i}+\Delta v\right) .
$$

Using this new function, we can write the Boltzman Eq. (20) as

$$
\frac{d f}{d t}=\left.\frac{\partial \tilde{f}_{i}}{\partial t}\right|_{\Delta r, \Delta v}+\frac{\partial \tilde{f}_{i}}{\partial \Delta r} \cdot \Delta v-\frac{\partial \tilde{f}_{i}}{\partial \Delta v} \cdot(\Delta r \cdot \nabla) \nabla \Phi=0 .
$$

Notice that Eq. (22) can be understood as the tidal limit of the Boltzmann equation in terms of the phase coordinates $(\Delta r, \Delta v)$, i.e. in the coordinate system comoving with particle $i$.

The stable clustering hypothesis assumes that $\left.\frac{\partial \tilde{f}_{i}}{\partial t}\right|_{\Delta r, \Delta v}$ averaged over the particles vanishes for small $\Delta r$ and $\Delta v$. This implies that the number of neighbors within a fixed physical separation of a DM particle in the phase space does not vary with time. Now, if we assume that $\left\langle\tilde{f}_{i} \nabla \nabla \Phi\right\rangle_{p} \approx\left\langle\tilde{f}_{i}\right\rangle_{p}\langle\nabla \nabla \Phi\rangle_{p}$, then a solution to Eq. (22) is

$$
\langle\tilde{f}\rangle_{p} \equiv \frac{1}{N} \sum_{i} \tilde{f}_{i}=F\left[\Delta v^{2}+\Delta x_{j} \Delta x_{k}\left\langle\partial_{j} \partial_{k} \Phi\right\rangle_{p}\right]
$$

where $F$ is the general solution with isotropic velocity distribution and $N$ is the number of particles in the phase-space volume of interest. By using the approximation of a spherically symmetric potential, the above solution can be rewritten by applying the Poisson equation:

$$
\langle\tilde{f}\rangle_{p}=\mu \xi_{s}=F\left[(\Delta v)^{2}+100 H^{2}\left(\xi_{s}\right)(\Delta r)^{2}\right],
$$

where $\xi_{s}$ and $H\left(\xi_{s}\right)$ are the phase-space density and Hubble constant at the formation time of DM substructure, respectively (see Fig. 1). We also use the spherical collapse model prediction for the halo density, which is roughly 200 times the critical density at the formation time [22]. $\mu \simeq 3 \%$ is the mean fraction of bound particle pairs that can survive the tidal disruption period, and is calibrated by comparison with $N$-body simulations [17]. To determine the function $F$, we use the spherical collapse model results. The phase-space density can be expressed as

$$
\xi_{s} \sim \frac{10 H\left(\xi_{s}\right)}{G^{2} M\left(\xi_{s}\right)},
$$

using the fact that the radius and velocity dispersion of halos are related as

$$
\sigma_{\text {vir }} \sim 10 H r_{\text {vir }} .
$$

The phase-space volume of the collapsed halo, i.e. the volume of the constant- $\xi_{s}$ ellipsoid in Eq. (23), is $M / \xi_{s}$, and by using Eq. (25), we find

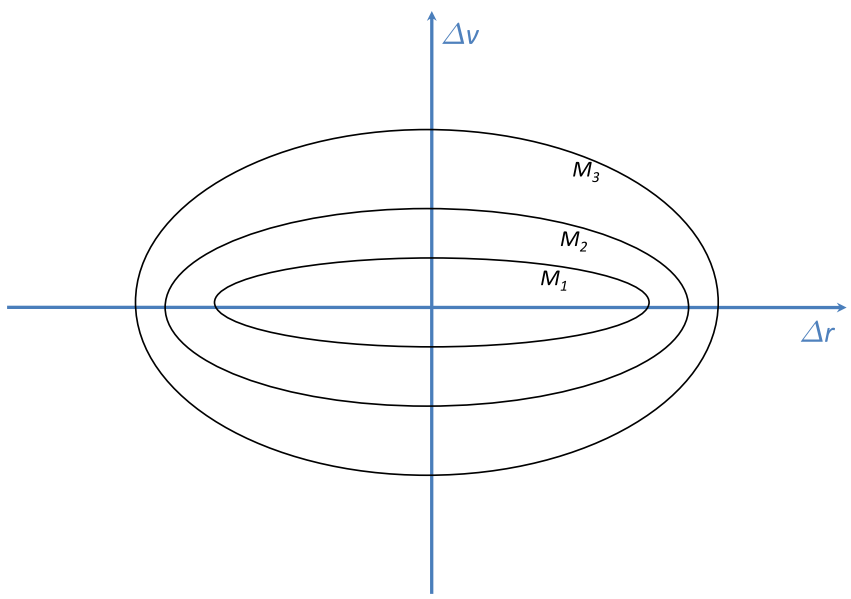

FIG. 1 (color online). Surfaces of constant average CDM phase-space density, $\langle\tilde{f}\rangle_{p}=\mu \xi_{s}$, around a typical particle in the stable clustering hypothesis. The surfaces are assumed to be concentric ellipsoids (Eq. (24)). The mass and Hubble scales at the collapse of the structure, $M\left(\xi_{s}\right)$ and $H\left(\xi_{s}\right)$, are related to the phase-space density, $\mu \xi_{s}$, on each surface via the spherical collapse results Eqs. (25)-(28), while $\mu \sim 3 \%$ is an empirical factor that quantifies tidal stripping and is fixed through comparison with numerical simulations [17]. 


$$
\left[\frac{\pi F^{-1}\left(\mu \xi_{s}\right)}{10 H\left(\xi_{s}\right)}\right]^{3}=\frac{\left[G M\left(\xi_{s}\right)\right]^{2}}{10 H\left(\xi_{s}\right)} .
$$

Furthermore, the mass scale that collapses at a given cosmological epoch is characterized by

$$
\left[\frac{H\left(\xi_{s}\right)}{H_{0}}\right]^{-2 / 3} \sigma\left[M\left(\xi_{s}\right)\right] \sim \delta_{c} \simeq 1.7
$$

where $\delta_{c}$ is the linear density threshold for the spherical collapse, $\sigma[M]$ is the rms top-hat linear over density at the mass scale $M$, and $H_{0}$ is the Hubble constant in the present epoch. Using the above result, the phase-space correlation function is obtained as

$$
\begin{aligned}
\left\langle f\left(r_{1}, v_{1}\right) f\left(r_{2}, v_{2}\right)\right\rangle & \\
& \simeq \frac{1}{V_{6}} \int_{V_{6}} d^{3} r d^{3} v f(r, v) f(r+\Delta r, v+\Delta v) \\
& =\frac{1}{V_{6}} \sum_{i} f\left(r_{i}+\Delta r, v_{i}+\Delta v\right) \\
& =\frac{N}{V_{6}}\langle\tilde{f}\rangle_{p} \\
& \simeq\left\langle f\left(r_{1}, v_{1}\right)\right\rangle\left\langle f\left(r_{2}, v_{2}\right)\right\rangle+\mu\langle f(\bar{r}, \bar{v})\rangle \xi_{s}(\Delta r, \Delta v) .
\end{aligned}
$$

In the equation above we used the assumption of ergodicity to replace the ensemble average \langle\rangle by the volume average, in a given volume of phase space $V_{6}$, while $(\bar{r}, \bar{v})$ are the mean values of $\left(r_{1}, v_{1}\right)$ and $\left(r_{2}, v_{2}\right)$. The second term is based on the stable clustering described above, with the assumption that $|\triangle v|=\left|v_{1}-v_{2}\right| \ll \Delta v_{\text {tid }}$ and $|\triangle r|=$ $\left|r_{1}-r_{2}\right| \ll \Delta r_{\text {tid }}$ where $\Delta v_{\text {tid }}$ and $\Delta r_{\text {tid }}$ characterize the tidal truncation radii in the phase space. On the other hand, the first term in Eq. (29) dominates for large separations in the phase space, where particles are not correlated. So Eq. (29) is an interpolation between the stable clustering and the smooth halo regimes. This is a crucial point in calculating the nonlinear power spectrum of structures on small scales where it is related to phase-space density correlation $\mu\langle f(\bar{r}, \bar{v})\rangle \xi_{s}(\Delta r, \Delta v)$ term.

\section{PULSAR RESIDUAL POWER SPECTRUM FROM STABLE CLUSTERING HYPOTHESIS}

In order to calculate the dimensionless power spectrum of pulsar frequency change, we need to know the power spectrum of matter on small scales. We now make use of the stable clustering hypothesis prediction, as developed in the previous section.

To use the stable clustering formula obtained in Eq. (29), we must relate the matter density power spectrum in Eqs. (13) and (19) to the real space correlation function of densities. In the stable clustering hypothesis in phase space, on small scales this relation becomes

$$
\begin{aligned}
\left\langle\rho\left(\vec{r}_{l}\right) \rho\left(\vec{r}_{l l}\right)\right\rangle & =\int d^{3} \vec{v}_{l} d^{3} \vec{v}_{l l}\left\langle f\left(\vec{r}_{l}, \vec{v}_{l}\right) f\left(\vec{r}_{l}, \vec{v}_{l l}\right)\right\rangle \\
& \simeq \int d^{3} \overline{\vec{v}} d^{3} \Delta \vec{v} \mu\langle f(\overrightarrow{\bar{r}}, \overrightarrow{\bar{v}})\rangle \xi_{s}(\Delta r, \Delta v) \\
& =\mu \bar{\rho}_{\text {avg }} \int d^{3} \Delta \vec{v} \xi_{s}(\Delta r, \Delta v) .
\end{aligned}
$$

In order to find the dependence of the dimensionless power spectrum $\omega P_{((\delta \nu) /(\nu))}(\omega)$ on $\omega$, we should have the rms tophat linear overdensity $\sigma(M) . \sigma(M)$ is the integral of linear matter power spectrum on a chosen window function as

$$
\sigma^{2}(M)=\int \frac{d^{3} k}{(2 \pi)^{3}} P_{L}(k) W^{2}(k R),
$$

where $P_{L}(k)$ and $W(k R)$ are the linear matter power spectrum and the Fourier transform of the spherical top-hat filter of radius $R$, respectively, where

$$
\begin{gathered}
P_{L}(k)=A k^{n_{s}} T^{2}(k), \\
W(x)=\frac{3(\sin x-x \cos x)}{x^{3}} .
\end{gathered}
$$

Here $n_{s}$ is the scalar spectral index of primordial matter power spectrum and $M=4 \pi R^{3} \bar{\rho}_{m} / 3$. The transfer function can be approximated by the BBKS [23] fitting formula,

$$
\begin{aligned}
T(k= & \left.q \Omega_{m} h^{2} \mathrm{Mpc}^{-1}\right) \\
\approx & \frac{\ln [1+2.34 q]}{2.34 q}\left[1+3.89 q+(16.2 q)^{2}\right. \\
& \left.+(5.47 q)^{3}+(6.71 q)^{4}\right]^{-1 / 4} .
\end{aligned}
$$

Using Eqs. (30) and (31), and the stable clustering hypothesis, we can find an expression for the matter power spectrum on small scales, shown in Fig. 2 for a mass range $10^{-6}$ to $10^{12}$ solar masses and $\mu=0.03$. For qualitative comparison, we also plot the dimensionless power spectrum $\Delta^{2}(k)=\frac{k^{3}}{2 \pi^{2}} P_{\mathrm{NL}}(k)$ obtained from the halo model of structure formation [24], as well as the fitting formulas of Peacock and Dodds [16] and Smith et al. for the nonlinear power spectrum [25]. We note that these approximations are based on fits to numerical simulations at $k \lesssim 10^{2} \mathrm{Mpc}^{-1}$, while the stable clustering hypothesis (which goes into second term in Eq. (29)) is expected to hold for $k \gg 10 \mathrm{Mpc}^{-1}$, and thus should be a more appropriate measure of small scale dark matter structures. On larger scales, the matter power spectrum is dominated by the first term on Eq. (29), which is equivalent to the standard halo model (i.e. the long-dashed line in Fig. 2). The cutoff in the halo model power spectrum is related to the size of smallest halo mass of $M_{\min }=10^{-6} M_{\odot}$.

Now, using the nonlinear power spectrum obtained from stable clustering, the dimensionless power spectrum for the Shapiro time-delay effect can be written as 


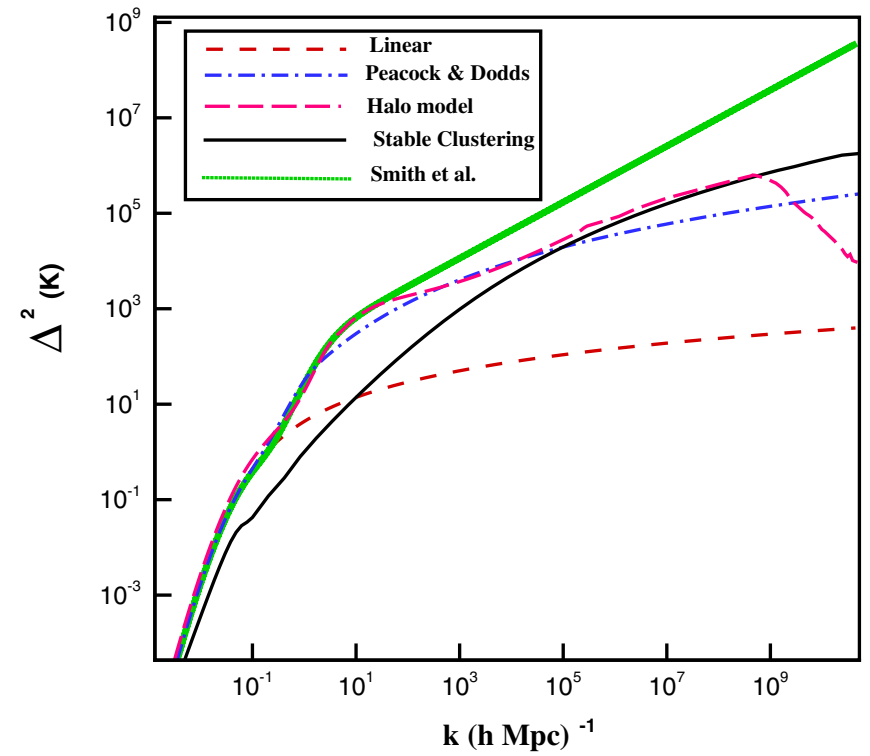

FIG. 2 (color online). Dimensionless power spectrum of density fluctuations $\Delta^{2}(k)=\frac{k^{3} P_{\mathrm{NL}}(k)}{2 \pi^{2}}$ as a function of wave number $k$ for the linear regime (short-dashed line), Peacock and Dodds fitting formula (dash-dotted line), Smith et al. fitting formula (thick dotted line), halo model (long-dashed line) and for $k \lessgtr$ $10^{2}$ stable clustering hypothesis used in this work (solid line).

$$
\begin{aligned}
& \left.\omega P_{((\delta v) /(\nu))}(\omega)\right|_{\text {Shapiro }} \\
& =\frac{4 z_{0}}{v} \mu \bar{\rho}_{\text {halo }} \int \frac{d k_{y}}{2 \pi} \omega^{3} \frac{(4 \pi G)^{2}}{k^{4}} \\
& \quad \times \int 4 \pi(\Delta r)^{2} d(\Delta r) \frac{\sin (k \Delta r)}{k \Delta r} \\
& \quad \times \int d(\Delta v) 4 \pi(\Delta v)^{2} \frac{10 H\left[\xi_{s}(\Delta r, \Delta v)\right]}{G^{2} M\left[\xi_{s}(\Delta r, \Delta v)\right]} .
\end{aligned}
$$

In the case of the Doppler effect, we can also find the dimensionless power spectrum of pulsar frequency change in terms of phase-space density derived from the stable clustering hypothesis. In this case Eq. (19) is expressed as

$$
\begin{aligned}
& \left.\omega P_{((\delta v) /(\nu))}(\omega)\right|_{\text {Doppler }} \\
& =\frac{\mu \bar{\rho}_{\text {halo }}}{v} \int \frac{d k_{y}}{4 \pi} \frac{k_{y}^{3}}{\omega} \frac{(4 \pi G)^{2}}{k^{4}} \\
& \quad \times \int 4 \pi(\Delta r)^{2} d(\Delta r) \frac{\sin (k \Delta r)}{k \Delta r} \\
& \quad \times \int d(\Delta v) 4 \pi(\Delta v)^{2} \frac{10 H\left[\xi_{s}(\Delta r, \Delta v)\right]}{G^{2} M\left[\xi_{s}(\Delta r, \Delta v)\right]} .
\end{aligned}
$$

Notice that $k=\sqrt{\left(\frac{\omega}{v}\right)^{2}+k_{y}^{2}}$, and the Hubble parameter and the mass are related by $\sigma(M)$ through Eq. (28). $\bar{\rho}_{\text {halo }}$ is the smoothed halo local density at solar system which is assumed to be $\sim 10^{5} \rho_{\text {crit }}$.

In order to numerically perform the third integrations in Eqs. (35) and (36) over $\left(k_{y}, \Delta r, \Delta v\right)$, we trade $\Delta v$ with $M$ as the integration variable using $F^{-1}=(\Delta v)^{2}+$ $100 H^{2}(\Delta r)^{2}$ (see Fig. 1). We then perform the integration in three steps:

(1) Noting that fixing $M$ and $\Delta r$ fixes $\Delta v$ through Eqs. (24)-(28), we can first perform the $k_{y}$ integral for fixed $\Delta r$ and $\Delta v$. Since the integrand can have fast oscillations in $k_{y}$, we find asymptotic expansions for the $k_{y}$ integral in the $\omega \Delta r / v \gg$ and $\ll 1$ limit, and devise an interpolation between the two regimes with less than $1 \%$ error, compared to the exact integral.

(2) We then perform the $\Delta r$ integral from zero to the maximum of $\left(F^{-1}\right)^{1 / 2} /(10 H)$, which is fixed by mass $M$, through Eq. (24).

(3) Finally, we take the integral over substructure mass, $M$, from $M_{\min }$ to $M_{\max }$, which we discuss below. We note that $H\left[\xi_{s}(\Delta r, \Delta v)\right]$ also becomes a function of $M$.

Now we are able to calculate the dimensionless power spectrum $\omega P_{((\delta \nu) /(\nu))}(\omega)$ in terms of the frequency $\omega$ numerically, for Shapiro time delay and the Doppler effect. For convenience we define the dimensionless parameter $h_{p}$ as

$$
h_{p} \equiv\left[\frac{1}{2 \pi} \omega P(\omega)\right]^{(1 / 2)},
$$

which is shown in Fig. 3 for the Doppler effect (solid line), Shapiro delay (dot-dashed line), and white noise (dashed line, which is computed in the Appendix). In order to calculate $h_{p}$ numerically, we consider a realistic set of parameters (but later study the effect of changing these parameters). We choose the velocity of dark matter substructures $v=300 \mathrm{~km} / \mathrm{s}$ (typical of relative velocities in the Milky Way halo), the typical distance of pulsars to $z_{0}=1 \mathrm{kpc}$, the mean fraction of bound particles that can survive the tidal disruption period $\mu=0.03$ [17], the minimum mass of DM substructure $M_{\min }=10^{-6} M_{\odot}$ and also the maximum $M_{\max }=10^{12} M_{\odot}$ (the total mass of a galactic halo). Later we will show that $h_{p}$ is almost independent of $M_{\max }$.

The power spectra of Shapiro and Doppler effects in Fig. 3 are well described by power laws:

$$
\begin{aligned}
& \left.\omega P_{((\delta \nu) /(\nu))}(\omega)\right|_{\text {Shapiro }} \propto \omega^{-3}, \\
& \left.\omega P_{((\delta \nu) /(\nu))}(\omega)\right|_{\text {Doppler }} \propto \omega^{-4} .
\end{aligned}
$$

These behaviors can be understood by noticing that the $\Delta v$ integral (i.e. the last integral) in Eqs. (35) and (36) scales as $H^{2}\left(\xi_{s}\right)$, if we use the spherical collapse relations of Sec. III. Since most small structures with CDM initial conditions collapse around the same time, this is approximately constant. The contribution to the rest of the integrals is dominated by $k_{y}^{-1} \sim \Delta r \sim v / \omega$, so the integral 
Timescale (year)

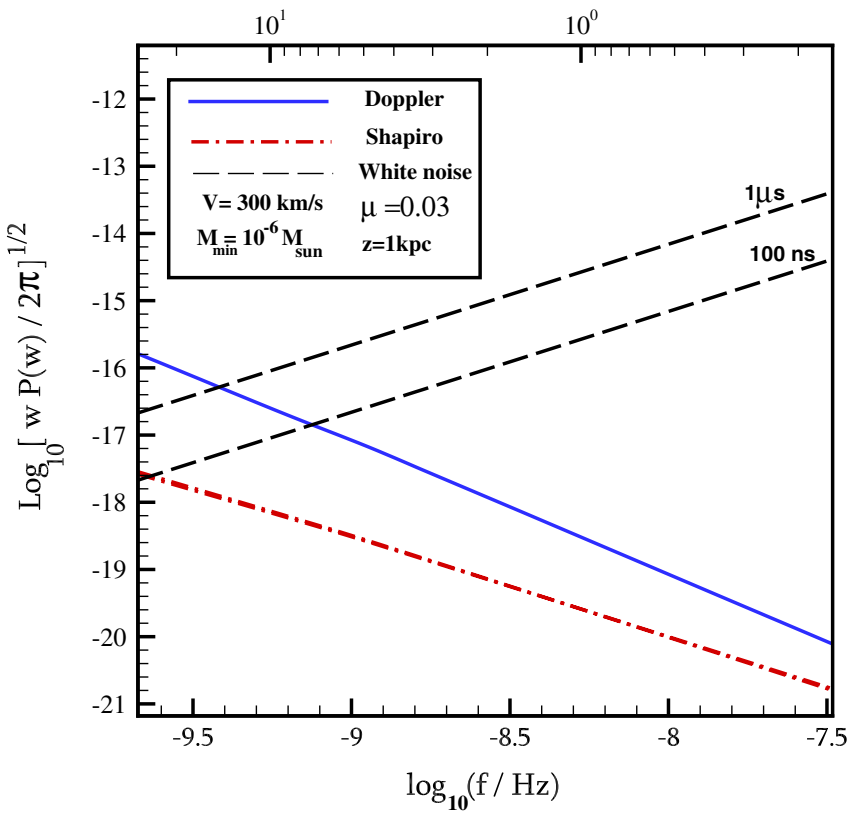

FIG. 3 (color online). Pulsar residual power spectrum as a function of frequency (bottom $x$-axis) and the span of observation time in years (top $x$-axis) for time delay caused by the Doppler effect (solid line) and time delay caused by Shapiro effect (dash-dot line). The long dashed lines represent levels of white noise for $100 \mathrm{~ns}$ (bottom) and $1 \mu \mathrm{s}$ (top) measured biweekly (see the Appendix) [18].

over distances scales as $(\Delta r)^{3} \propto \omega^{-3}$. Plugging this into Eqs. (35) and (36) yields the scalings of Eq. (38).

To physically understand the scaling for the Doppler effect we can once more Fourier transform the powerspectrum in Eq. (36) to find that $v_{\text {Dop. }} \sim \frac{\delta \nu}{\nu}$ is proportional to $v t^{2}=(v t) \times t$, i.e. the magnitude of acceleration is proportional to distance traveled by the earth/pulsar. This is exactly what one expects for the gravitational field in a medium with roughly uniform density, and is due to the fact that most small substructure forms at roughly the same density $\propto H^{2}\left(\xi_{s}\right)$. However, the direction of acceleration is random, as different substructures will dominate the local gravity on different scales.

An important point to consider before examining the effect of different parameters on pulsar timing is the study of the effect of maximum mass in the integrals. As we show in Fig. 4 , the total dependence of $h_{p}$ on maximum mass is small, where we plot the $h_{p}$ for $M_{\max }=10^{12} M_{\odot}$, the total mass of a typical galaxy and $M_{\max }=10^{8} M_{\odot}$, for a more realistic tidal cut-off for subhaloes at our position in the Milky Way. This confirms that, not surprisingly, most of the observable effects on pulsar timing comes from CDM small scale structure.

Now we examine the dependence of the power spectrum on different parameters of the model. We plot the dimensionless amplitude $h_{p}$ for the Doppler effect for different

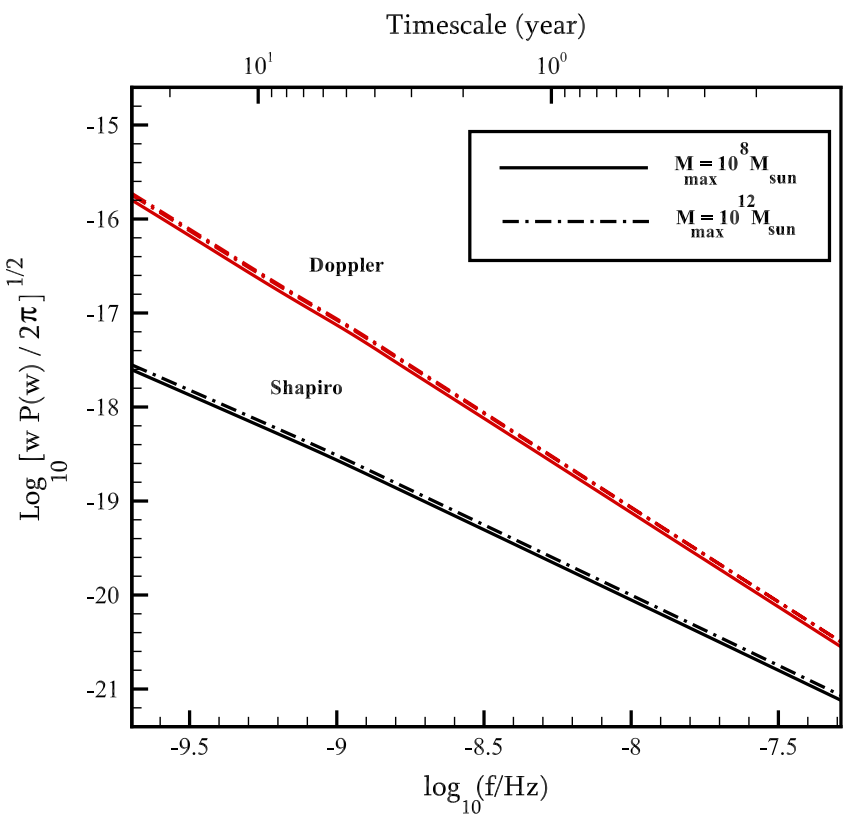

FIG. 4 (color online). Pulsar residual power spectrum as a function of frequency (bottom- $x$ axis) and the span of time in years (top $x$-axis) for time delay caused by the Doppler effect (top line) and time delay caused by Shapiro effect (bottom line) for a maximum mass of halo $M_{\max }=10^{12} M_{\odot}$ (dash-dot line) and $M_{\max }=10^{8} M_{\odot}$ (solid line $)$.

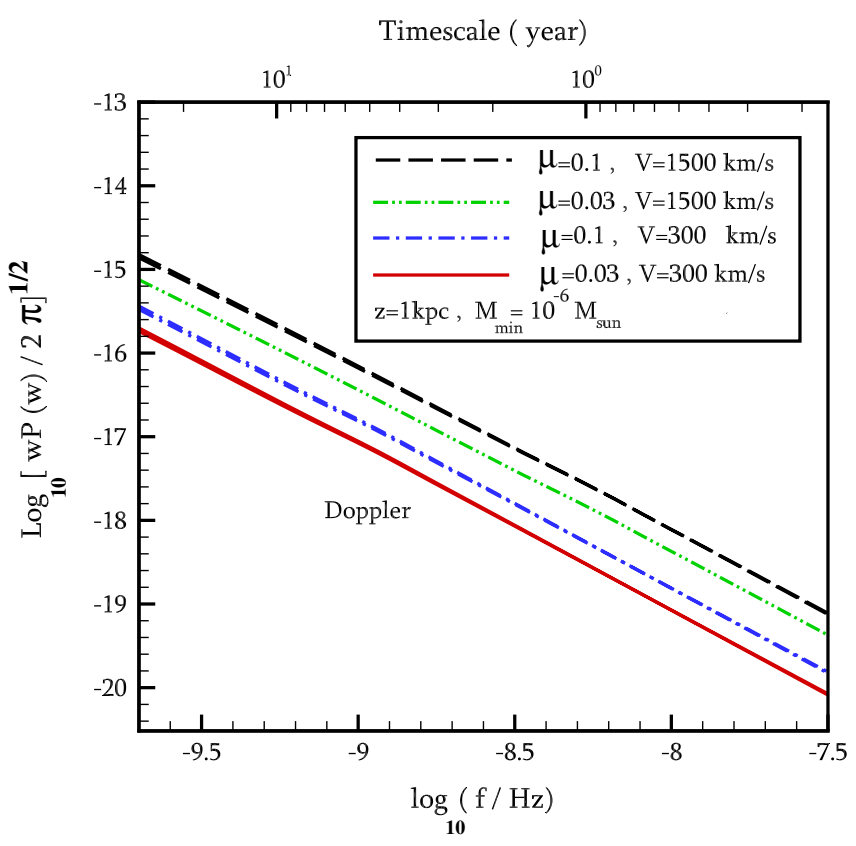

FIG. 5 (color online). Pulsar residual power spectrum as a function of frequency (bottom- $x$ axis) and the span of time in years (top $x$-axis) for Doppler effect (solid line) for different velocities and $\mu$ of dark matter substructures. 
Timescale ( year)

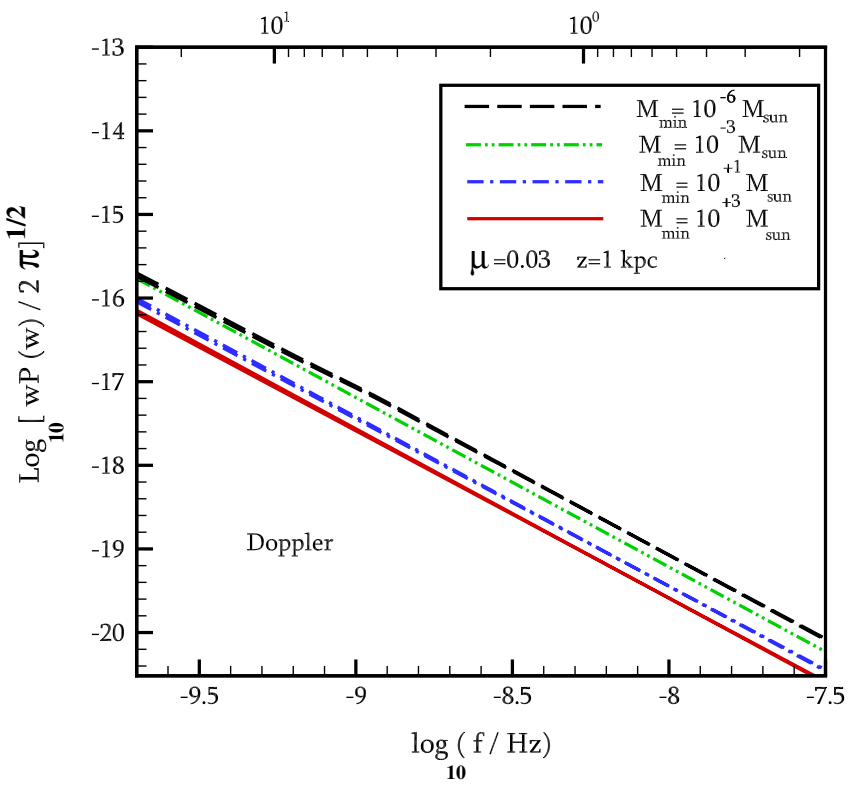

FIG. 6 (color online). Pulsar residual power spectrum as a function of frequency (bottom- $x$ axis) and the span of time in years (top $x$-axis) for Doppler effect (solid line) for different minimum masses of dark matter substructures.

velocities of dark matter substructures and the $\mu$-parameter of stable clustering in Fig. 5, which shows that $h_{p}$ is proportional to velocity and the square root of the $\mu$ parameter.

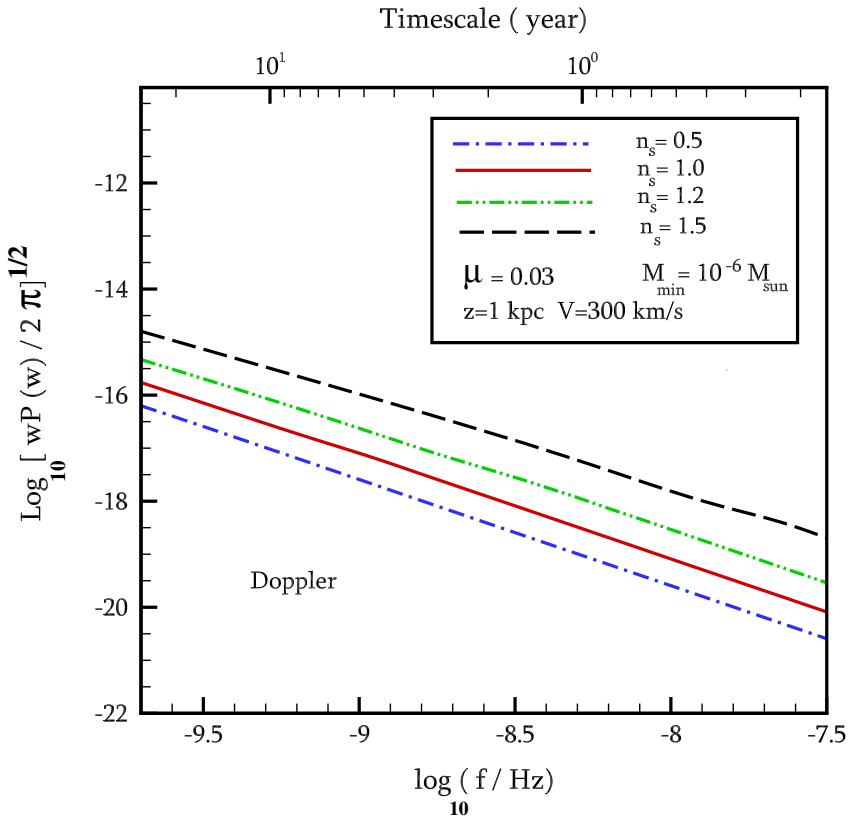

FIG. 7 (color online). Pulsar residual power spectrum as a function of frequency (bottom- $x$ axis) and the span of time in years (top $x$-axis) for Doppler effect for different primordial index of matter power spectrum.

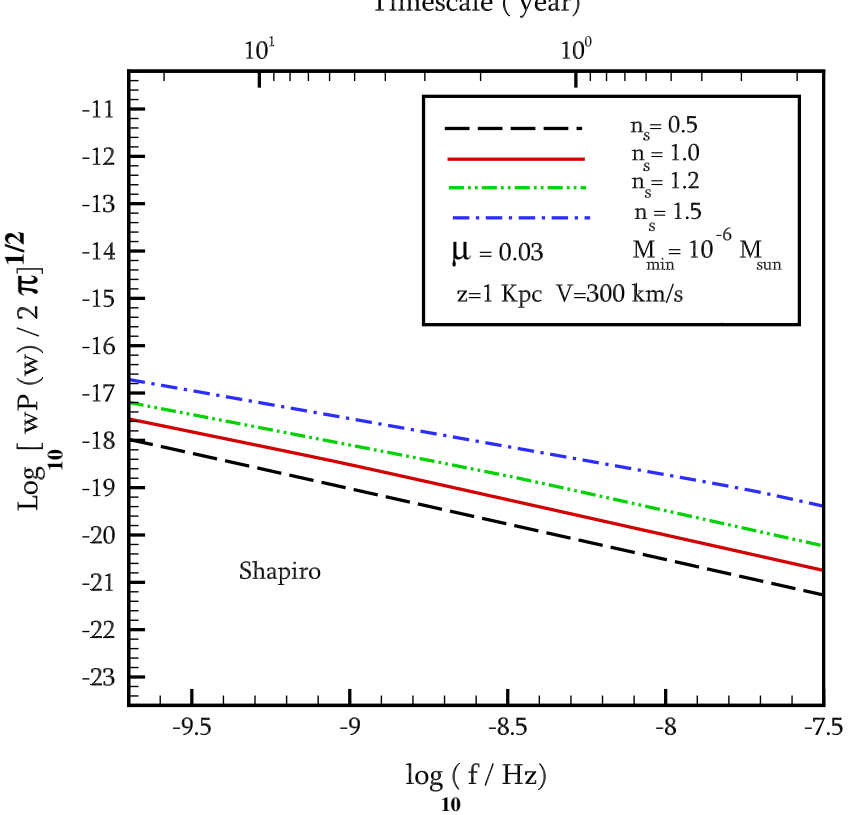

FIG. 8 (color online). Pulsar residual power spectrum as a function of frequency (bottom- $x$ axis) and the span of time in years (top $x$-axis) for Shapiro effect for different primordial index of matter power spectrum.

In Fig. 6, we plot the power spectrum for different mass minima of DM substructures. As shown in Fig. 6, the $\omega^{-4}$ dependence of $h_{p}^{2}$ does not change by changing the minimum of the mass. However, the amplitude of the signal increases when the interval of integration is increased.

In Figs. 7 and 8 we plot $h_{p}$ given different primordial spectral index $n_{s}$, for Doppler and Shapiro effects, respectively. For $n_{s}<1$, the slope of $h_{p}$ does not change, as $\sigma(M)$ becomes flat for low masses. On the other hand for larger $n_{s}$, we see a shallower $\omega$ dependence for $h_{p}$ as there is more power on small scales.

\section{OBSERVATIONAL PROSPECTS}

Finally, to explore the observational prospects for the detection of pulsar frequency change due to dark matter substructures, we compare our results with the observational bounds put on detection of gravitational waves (GW) by pulsars. The observed quantities are similar in both cases, and the power spectrum of pulsar frequency change caused by Doppler or Shapiro effects is red similar to gravitational waves. That is, there is an excess power at low frequencies, or long time-scale correlations in residuals. The gravitational wave effect on pulsar timing is also in the $n \mathrm{~Hz}$ frequency range [20,26], similar to the substructure effect we are considering here.

In particular, the frequency change due to gravitational waves is roughly $\sim h_{i j}$, the amplitude of gravitational waves, which allows us to directly translate constraints on $h_{i j}$, to constraints on $\delta \nu / \nu$. Moreover, similar to the 
characteristic quadrupolar pattern that gravitational waves induce in pulsar timing residuals (e.g., [26]), the Doppler effect induces a dipolar pattern in the sky, which can be used to distinguish it from intrinsic changes in individual pulsars.

More specifically, the frequency shift due to the combination of Doppler effect, gravitational waves, and intrinsic effects is given by

$$
\left.\frac{\delta \nu(t)}{\nu}\right|_{a}=I_{a}(t)+\hat{\mathbf{n}}_{a} \cdot \mathbf{v}(t)+\frac{\hat{\mathbf{n}}_{a} \cdot \mathbf{h}(\mathbf{k}, t) \cdot \hat{\mathbf{n}}_{a}}{1+\hat{\mathbf{k}} \cdot \hat{\mathbf{n}}_{a}},
$$

where $\hat{\mathbf{n}}_{a}$ is the unit vector along the direction of pulsar $a$, $I_{a}(t)$ is the frequency shift intrinsic to the pulsar, $\mathbf{v}(t)$ is the earth's velocity, and $\mathbf{h}(\mathbf{k}, t)$ is the amplitude of a gravitational wave with wave-vector $\mathbf{k}$. The cross-power spectrum of frequency-change between different pulsars is given by

$$
\begin{aligned}
\left.P_{((\delta \nu) /(\nu))}(\omega)\right|_{a b}= & \left.P_{((\delta \nu) /(\nu))}(\omega)\right|_{\text {int. }} \delta\left(x_{a b}\right) \\
& +\left.P_{((\delta \nu) /(\nu))}(\omega)\right|_{\text {Doppler }}\left(1-2 x_{a b}\right) \\
& +\left.P(\omega)\right|_{\text {grav. }} c\left(x_{a b}\right),
\end{aligned}
$$

where

$$
x_{a b} \equiv\left(1-\hat{\mathbf{n}}_{a} \cdot \hat{\mathbf{n}}_{b}\right) / 2,
$$

and

$$
c(x) \equiv \frac{3}{2} x \ln x-\frac{x}{4}+\frac{1}{2},
$$

is the expected correlation pattern of timing residuals for an isotropic stochastic gravitational wave background [26]. Therefore, pulsar timing cross-power spectra are affected by the intrinsic, Doppler and gravitational waves, $\left.P_{((\delta \nu) /(\nu))}(\omega)\right|_{\text {int. }},\left.P_{((\delta \nu) /(\nu))}(\omega)\right|_{\text {Doppler }}$, and $\left.P(\omega)\right|_{\text {grav. }}$ with different angular dependences, which can be used to distinguish these effects.

In Fig. 9 we plot the realistic and optimistic predictions for detection of $h_{p}$, which is similar to the gravitational wave dimensionless strain, and compare it with the current observational limits from a pulsar timing array [19] considering the sensitivity limit for time residuals of observed millisecond pulsars obtained via (see the Appendix of [27] for details)

$$
h_{p}^{\lim } \propto \frac{\delta t_{\mathrm{rms}} f}{N_{p}^{1 / 2}(T \Delta f)^{1 / 4}},
$$

where $h_{p}^{\lim }$ is the sensitivity limit of detectors, $\delta t_{\mathrm{rms}}=$ $\sqrt{\left\langle\delta t^{2}\right\rangle}$ is the root mean square value of the timing residuals, $\Delta f$ is the frequency bandwidth of search, $N_{p}$ is the number of pulsars, and $T$ is the time span of observation. The pulsar timing array sensitivity is scaled with frequency as $h_{p}^{\lim } \propto f$ and reaches a minimum at a detectable frequency of $f \sim 1 / T$. This produces the wedgelike sensitivity limit curves in Fig. 9. The sensitivity limit is also

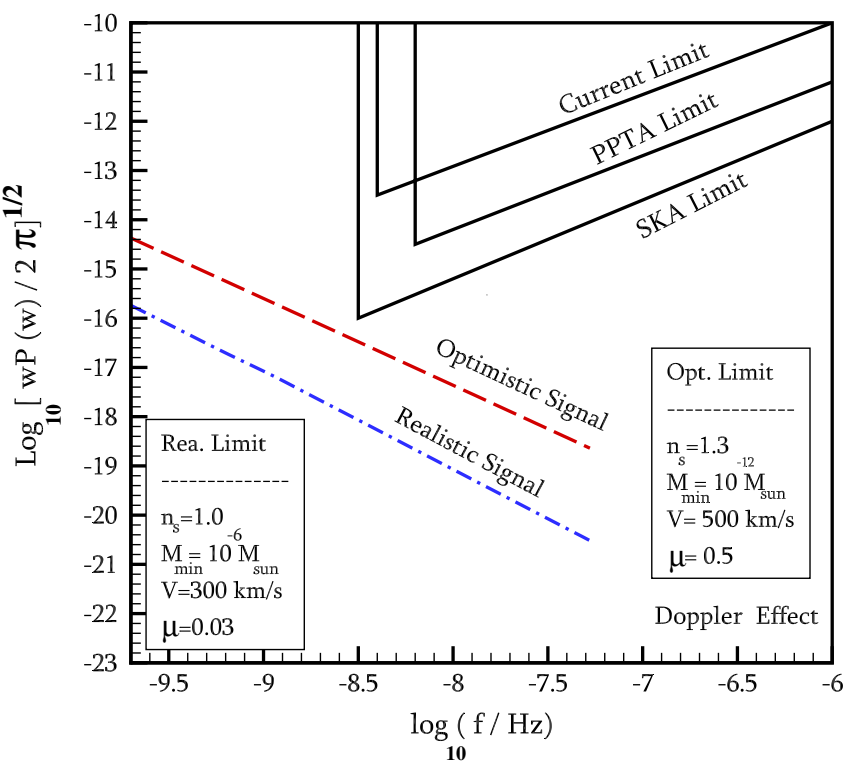

FIG. 9 (color online). Pulsar residual power spectrum as a function of the frequency for Doppler effect for realistic and optimistic signals (see text for definition of realistic and optimistic parameters). The limits from current and future experiments are also shown.

proportional to $\delta t_{\mathrm{rms}}$, improving as the precision of pulsar timing residuals detection is increased. By increasing the observational time, we increase the sensitivity and also the span of frequency.

We also plot the predicted sensitivity of Parkers pulsar timing array (PPTA) [28] and the square kilometer array (SKA) [29] for $h_{p}$. The upper bounds for future PPTA and SKA experiments are obtained from the detectable time residual correlation of simulated pulsars with consideration of all instrumental, calibration and observational errors (such as pulsar intrinsic period changes and glitches) [30]. For example the PPTA bound is obtained by considering 20 radio pulsars for 5 years with $\delta t_{\mathrm{rms}}=100 \mathrm{~ns}$ which provides a peak sensitivity of $h_{p}^{\lim } \approx 2 \times 10^{-15}$ at $f \approx 7 \times 10^{-9}$. For SKA, with the same number of pulsars, the sensitivity is improved by increasing the span of observation to 10 years with timing accuracy $\delta t_{\mathrm{rms}}=10 \mathrm{~ns}$, leading to a constraint on the pulsar residual power spectrum of $\sim 1.6 \times 10^{-16}$ at $f \approx 7 \times 10^{-9}$ [27].

Finally, we study the effect of uncertainty in the models of nonlinear structure formation on our results. In other words, how much will our resultsdepend on the choice of stable clustering hypothesis? As we argued above, stable clustering is the only known physical prediction for the nonlinear power spectrum on very small scales. Nevertheless, we can calculate $h_{p}$ for the nonlinear power spectra of other clustering models in Fig. 2. The relative magnitude of $h_{p}$ in two different models is obtained from Eq. (19): 


$$
\frac{h_{p}^{(m 1)}}{h_{p}^{(m 2)}}=\left[\left(\int d k_{*} \frac{k_{*}^{3}}{k^{4}} P_{\mathrm{NL}}^{(m 1)}(k)\right) /\left(\int d k_{*} \frac{k_{*}^{3}}{k^{4}} P_{\mathrm{NL}}^{(m 2)}(k)\right)\right]^{1 / 2},
$$

where superscript $(m 1)$ and $(m 2)$ indicate the models. In Fig. 10, we plot $h_{p}$ for different models of nonlinear structure formation by using the realistic parameters for the models.

An interesting point to notice is that different models of nonlinear structure formation have (almost) the same frequency dependence, $\omega P_{\delta \nu / \nu} \propto \omega^{-4}$, as in stable clustering. This is because of the moving screen approximation $k_{x} v=\omega$ and $k_{z} \sim z^{-1} \ll k_{x}, k_{y}$, which is applicable in the alternative models as well. On the other hand, the main contribution of the integrals in Eq. (44) from the nonlinear matter power spectrum comes when $k_{y} \sim$ $\omega / v \sim 10^{-8} \mathrm{~Hz} / 300 \mathrm{~km}-10^{9} \mathrm{Mpc}^{-1}$. In this case $h_{p}^{m 1} / h_{p}^{m 2}$ reduces to the ratio of $P_{\mathrm{NL}}$ 's, which is nearly independent of wave number (and thus frequency; see Fig. 2) for relevant scales.

Closer examination indicates that the frequency dependence of the Smith et al. model is slightly shallower than the others $\left(h_{p}^{\text {smith }} \sim \omega^{-1.93}\right)$. This is due to the fact that Smith et al. predict a much bluer spectrum on small scales (Fig. 2), which is similar to the case of stable clustering with higher power index (Fig. 7).

In summary, we find that the signature of the Doppler effect in pulsar timing is largely independent of the nonlinear structure formation model, which only introduces (a factor of a few) uncertainty in the amplitude of timing residuals, $h_{p}$. Our results show that while current

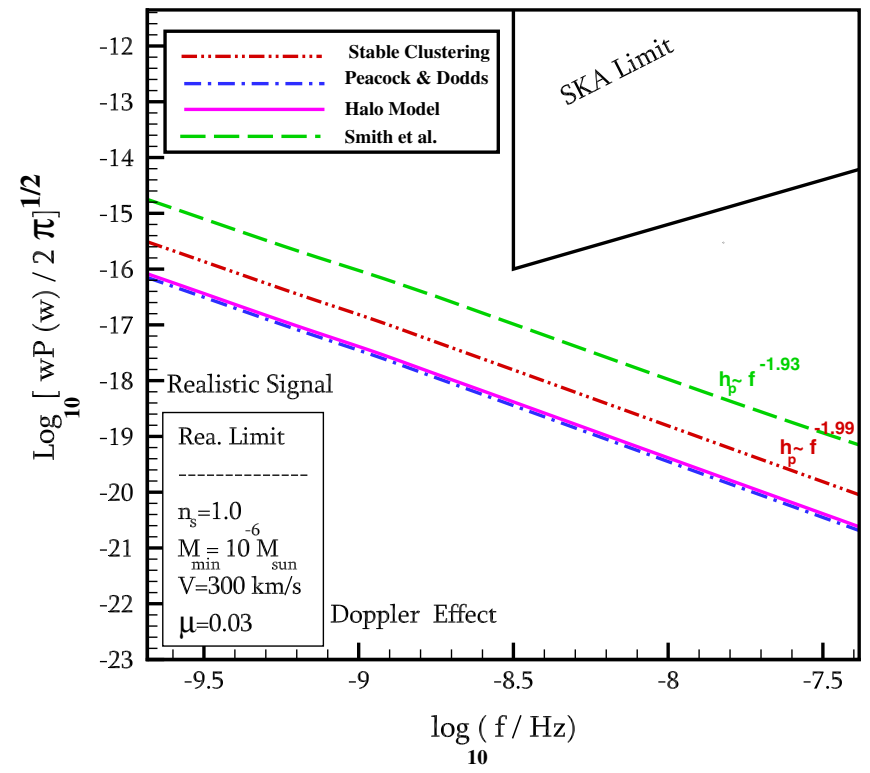

FIG. 10 (color online). Pulsar residual power spectrum as a function of the frequency due to Doppler effect, for realistic signals in different models of nonlinear structure formation. observations are unable to detect the effect of dark matter substructure on pulsar timing, error projections for the upcoming square kilometer array (SKA) are only a factor of few higher than our optimistic predictions.

In the end, we should note that, unlike the Doppler effect, the Shapiro time delay does not have a coherent pattern on the sky, as different lines of sight are largely uncorrelated. This makes it much harder to distinguish Shapiro time delay from pulsar intrinsic frequency changes.

\section{CONCLUSIONS AND DISCUSSION}

In this work, we studied the gravitational effect of DM substructures on pulsar timing, through Doppler and Shapiro (or ISW) effects. We calculated the dimensionless power-spectrum of a pulsar's frequency-change, which is related to the matter density power-spectrum in the nonlinear regime. We used the stable clustering hypothesis to extract the nonlinear matter power-spectrum, and showed that the frequency-change is dominated by the Doppler effect. Next we varied the free parameters of the model, which had the following effects on the dimensionless power, $h_{p}$ :

(1) $h_{p}$ due to Doppler effect is linearly proportional to velocity of DM substructures.

(2) The main contribution of DM substructures comes from the minimum mass in DM hierarchy: as we increase the domain of integration over DM subhalo masses, we get more signal.

(3) $h_{p}$ has a dependence on $\mu^{1 / 2}$, the fraction of particle pairs that remain bound in the stable clustering hypothesis.

(4) $h_{p}$ due to the Shapiro effect scales as the square root of distances to pulsars, as it depends on the integrated gravitational effect over the line of sight.

(5) For larger primordial spectral index, $n_{s}$, the frequency dependence of $h_{p}$ is shallower, because the main contribution of $h_{p}$ comes from low masses, where the power is increased. However, for $n_{s}<1$, the frequency dependence becomes independent of $n_{s}$.

(6) The frequency dependence of $h_{p}$ is nearly independent of nonlinear structure formation model, although its amplitude could change by a factor of a few.

Finally, we compared the dimensionless power spectrum of pulsar frequency change, for realistic and optimistic sets of parameters, with current and future pulsar timing experiments, designed for detection of gravitational waves. Our results show that our optimistic estimate of the $h_{p}$ signal is only a factor of a few smaller than the sensitivity of the planned square kilometer array (SKA), making this method a potentially promising avenue for the detection of DM substructure on very small scales. While this may sound 
too futuristic, it is worth noting that more dedicated pulsar timing follow-ups of pulsars discovered by SKA, as well as better noise removal techniques for ISM contamination of timing signals (e.g., [31]) will be able to potentially push down the noise below our conservative forecasts.

We should further note that, as can be seen in Fig. 10, if this signal is ever detected, there will be degeneracies between parameters that quantify the nature of DM and those of structure formation (in both linear and nonlinear regimes). Therefore, further study into the nature and properties of the signal (or independent observables) will be necessary to disentangle these degeneracies.

While our paper lays the groundwork for future statistical detection of dark matter substructure through pulsar timing, many practical challenges and theoretical uncertainties remain. Here we point out two, along with potential resolutions:

First, it is important to note that the observed Doppler effect in pulsar timing depends on the total gravitational acceleration, which can be contributed by nearby stars/ planets, in addition to local dark matter substructure. However, the gravitational pull of stars/planets on Earth can be calculated by knowing their masses and positions around Earth, and thus, in principle, can be computed and corrected for (e.g., [32]). Similar effects on the acceleration of pulsars will be uncorrelated for different pulsars, and thus can be distinguished from Earth's acceleration.

A second concern is the possible non-Gaussianity of the signal. For example, microlensing events due to stars in the Galactic halo could lead to large magnifications, but have very small optical depth, and thus happen rarely. Therefore, the power spectrum gives a very incomplete description of the observables in microlensing events. However, in contrast to magnification events that trace projected density, the gravitational effects on pulsar timing that we discuss here trace the integrated potential, which is much more smooth. Moreover, the small CDM substructure is much more diffuse than stars, which further reduces the skewness of the signal. Therefore, unlike microlensing events, the observed signal is likely to be contributed by a variety of structures on different scales (e.g. Fig. 6) with no sharp boundaries. This is why we expect a close to Gaussian signal, simply based on the central limit theorem, which suggests that the power spectrum might provide adequate statistical description of these effects.

\section{ACKNOWLEDGMENTS}

We would like to thank Latham Boyle, Adrienne Erickcek, Sohrab Rahvar, and Ethan Siegal for discussion and valuable comments. S.B. thanks the Perimeter Institute for their kind hospitality during a visit where part of this work began. N. A. is supported by Perimeter Institute (PI) for Theoretical Physics and Natural Sciences and Engineering Research Council of Canada (NSERC). Research at PI is supported by the Government of Canada through Industry Canada and by the Province of Ontario through the Ministry of Research \& Innovation.

\section{APPENDIX: STATISTICS OF $\sigma_{z}$ FOR STABILITY OF PULSARS AND WHITE NOISE CALCULATION}

The ability of a pulsar timing array to detect any delay in the received pulses to measure the dark matter halos substructures depends on the pulsar timing stability. Timing stability is related to how long the rms of timing residuals can be kept small, from which we can estimate the potential to detect Doppler and Shapiro effects. Statistical artifacts such as a large gap in data sampling, or a large variation in error-bar size, may prevent a reliable power spectrum of pulsar timing data. An alternative approach is $\sigma_{z}$ statistics, as described by e.g. Matsakis et al. [33]:

$$
\sigma_{z}(\tau)=\frac{\tau^{2}}{2 \sqrt{5}}\left\langle c_{3}^{2}\right\rangle^{1 / 2},
$$

where \langle\rangle denotes the average over subsets of the pulsar timing data, and $c_{3}$ is determined from a polynomial fit

$$
c_{0}+c_{1}\left(t-t_{0}\right)+c_{2}\left(t-t_{0}\right)^{2}+c_{3}\left(t-t_{0}\right)^{3}
$$

to timing residuals for each subset, and $\tau$ is the length of the subsets. In order to connect our theoretical calculations to the observed pulsar time residuals we should find a relation between $\sigma_{z}$ and the calculated power spectrum. From the polynomial fit to the timing residuals we find that

$$
\left.c_{3} \simeq \frac{1}{6} \frac{d}{d \tau} \Delta \ddot{t}\right|_{s} \simeq \frac{1}{6} \frac{d}{d \tau} \frac{\dot{\delta} \nu}{\nu},
$$

where we assume that the fitting procedure depends on $\left.\Delta t\right|_{s}$, which is coarse grained on the scale of $\tau$. The correlation function $c_{3}$ can be written as

$$
\left\langle c_{3}^{2}\right\rangle=\frac{1}{18}\left\{\left\langle\left(\frac{\dot{\delta} \nu}{\nu}\right)^{2}\right\rangle-\left\langle\left.\left.\left(\frac{\dot{\delta} \nu}{\nu}\right)\right|_{l}\left(\frac{\dot{\delta} \nu}{\nu}\right)\right|_{u}\right\rangle\right\} .
$$

Now, using Eqs. (9), (A1), and (A4), we obtain

$$
\sigma_{z}(\tau) \simeq \frac{\tau}{6 \sqrt{5}}\left\{\int_{0}^{(1 / \tau)} \frac{d \omega}{2 \pi} \omega^{2} P(\omega)[1-\cos (\omega \tau)]\right\}^{1 / 2} .
$$

In order to find the white noise corresponding to pulsar timing we derive the relation of the dimensionless power spectrum of pulsars with the sampling time and the uncertainty in the pulsar timing measurement. The cross correlation of time residuals of pulsar timing is related to the accuracy of measurement $t_{a}$ as

$$
\left\langle\delta t\left(t_{1}\right) \delta t\left(t_{2}\right)\right\rangle=\left(t_{a}\right)^{2} \delta_{t_{1} t_{2}},
$$

where $\delta_{t_{1} t_{2}}$ is the Kronecker delta and $\delta t$ is the time residual of pulsar timing related to frequency change as

$$
\delta t=\int \frac{\delta \nu}{\nu} d t
$$


The correlation of timing residuals can be approximated in the time span of $\tau$, which is the period of sampling as

$$
\left\langle\delta t\left(t_{1}\right) \delta t\left(t_{2}\right)\right\rangle \simeq\left(t_{a}\right)^{2} \tau \delta\left(t_{1}-t_{2}\right) .
$$

Now the power spectrum of time residuals is obtained as

$$
P_{\delta t}(\omega)=\int e^{-i \omega t}\left\langle\delta t\left(t_{1}\right) \delta t\left(t_{2}\right)\right\rangle d t=\tau t_{a}^{2},
$$

which yields the dimensionless power spectrum,

$$
h_{p}=\left[\frac{1}{2 \pi} \omega P_{((\delta \nu) /(\nu))}(\omega)\right]^{1 / 2}=\frac{\sqrt{\tau}}{\sqrt{2 \pi}} \omega^{3 / 2} t_{a} .
$$

To find the white noise lines in Fig. 3, we set the sampling time of pulsar timing $\tau$ to be 2 weeks and the accuracy of pulsar timing, $t_{a}$, to be $100 \mathrm{~ns}$ and $1 \mu \mathrm{s}$.
[1] N. Jarosik et al., Astrophys. J. Suppl. Ser. 192, 14 (2011).

[2] M. Roos, arXiv:1001.0316.

[3] A. Loeb and M. Zaldarriaga, Phys. Rev. D 71, 103520 (2005); S. Profumo, K. Sigurdson, and M. Kamionkowski, Phys. Rev. Lett. 97, 031301 (2006); G. D. Martinez, J. S. Bullock, M. Kaplinghat, L. E. Strigari, and R. Trotta, J. Cosmol. Astropart. Phys. 06 (2009) 014.

[4] D. A. Frail et al., Astrophys. J. 436, 144 (1994).

[5] I. I. Shapiro, Phys. Rev. Lett. 13, 789 (1964).

[6] E. R. Siegel, M. P. Hertzberg, and J. N. Fry, Mon. Not. R. Astron. Soc. 382, 879 (2007).

[7] N. Seto and A. Cooray, Astrophys. J. 659, L33 (2007).

[8] M. Pshirkov, A. Tuntsov, and K. A. Postnov, Phys. Rev. Lett. 101, 261101 (2008).

[9] T. Ishiyama, J. Makino, and T. Ebisuzaki, Astrophys. J. Lett. 723, L195 (2010).

[10] M. J. Longo, Phys. Rev. Lett. 60, 173 (1988).

[11] T. I. Larchenkova and S. M. Kopeikin, Astron. Lett. 32, 18 (2006).

[12] K. Ohnishi, M. Hosokawa, T. Fukushima, and M. Takeuti, Astrophys. J. 448, 271 (1995); T. I. Larchenkova and O. V. Doroshenko, Astron. Astrophys. 297, 607 (1995); M. A. Walker, Publ. Astron. Soc. Aust. 13, 236 (1996); M. Hosokawa, K. Ohnishi, and T. Fukushima, Astron. Astrophys. 351, 393 (1999); M. S. Pshirkov, M. V. Sazhin, and Yu. P. Ilyasov, Astron. Lett. 34, 397 (2008).

[13] A. Erickcek and N. M. Law, Astrophys. J. 729, 49 (2011).

[14] M. Davis and P. J. E. Peebles, Astrophys. J. Suppl. Ser. 34, 425 (1977).

[15] A. J.S. Hamilton, A. Matthews, P. Kumar, and E. Lu, Astrophys. J. 374, L1 (1991); B. Jain, H. J. Mo, and S. D. M. White, Mon. Not. R. Astron. Soc. 276, L25 (1995).
[16] J. A. Peacock and S. J. Dodds, Mon. Not. R. Astron. Soc. 280, L19 (1996).

[17] N. Afshordi, R. Mohayaee, and E. Bertschinger, Phys. Rev. D 81, 101301 (2010).

[18] J. P. W. Verbiest et al., Mon. Not. R. Astron. Soc. 400, 951 (2009).

[19] F. A. Jenet et al., Astrophys. J. 653, 1571 (2006).

[20] F. A. Jenet, G. B. Hobbs, K. J. Lee, and R. N. Manchester, Astrophys. J. 625, L123 (2005).

[21] R. K. Sachs and A.M. Wolfe, Astrophys. J. 147, 73 (1967).

[22] J. E. Gunn and J. R. Gott, Astrophys. J. 176, 1 (1972).

[23] J. M. Bardeen, J. R. Bond, N. Kaiser, and A. S. Szalay, Astrophys. J. 304, 15 (1986).

[24] A. Cooray and R. K. Sheth, Phys. Rep. 372, 1 (2002).

[25] R. E. Smith et al., Mon. Not. R. Astron. Soc. 341, 1311 (2003).

[26] G. Hobbs, F. A. Jenet, K. J. Lee, J.P. W. Verbiest, D. Yardley, R. Manchester, A. Lommen, W. Coles, R. Edwards, and C. Shettigara, Mon. Not. R. Astron. Soc. 394, 1945 (2009).

[27] A. Sesana, A. Vecchio, and C. N. Colacino, Mon. Not. R. Astron. Soc. 390, 192 (2008).

[28] R. N. Manchester, arXiv:1004.3602; G. B. Hobbs et al., PASA 26, 103 (2009); J. P. W. Verbiest et al., Classical Quantum Gravity 27, 084015 (2010).

[29] R. Smiths et al., arXiv:0811.0211.

[30] G. Hobbs, arXiv:1006.3969.

[31] P. Demorest, arXiv:1106.3345.

[32] D. J. Champion et al., Astrophys. J. 720, L201 (2010).

[33] D. N. Matsakis, J.H. Taylor, and T. M. Eubanks, Astron. Astrophys. 326, 924 (1997). 